
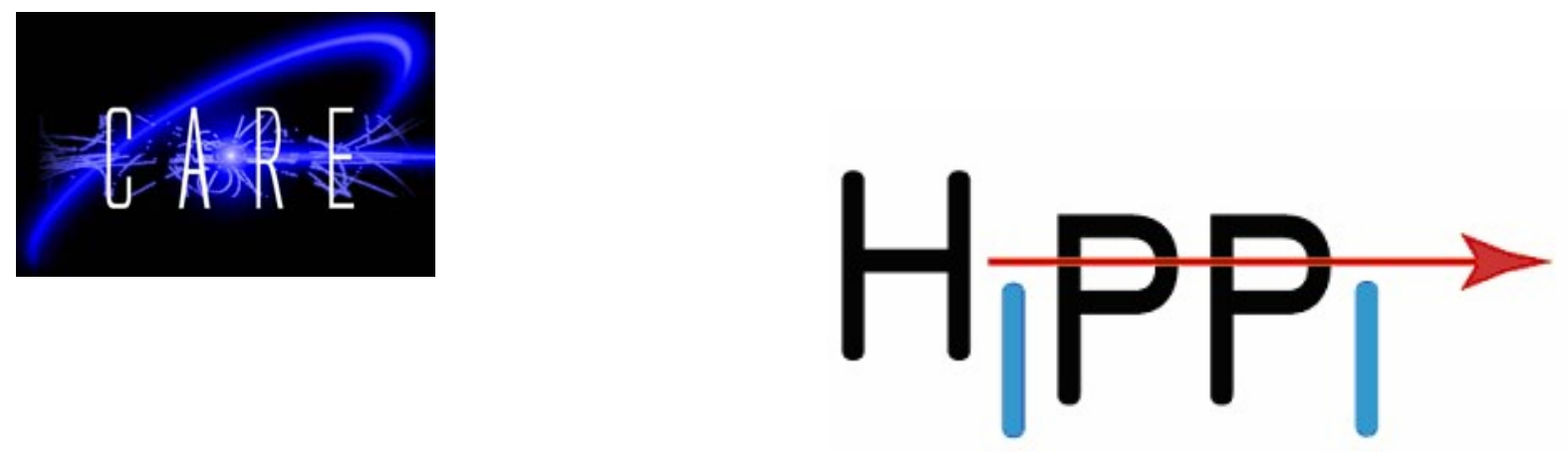

\title{
COMPARISON OF BEAM DYNAMIC IN DIFFERENT SUPERCONDUCTING OPTIONS OF LOW ENERGY HIGH INTENSE LINAC
}

N.Vasyukhin, Yu.Senichev and R.Tölle, Forschungszentrum Jülich, Germany

\begin{abstract}
At present the superconducting proton linacs have obvious applications in energy range $~ 100$ $1000 \mathrm{MeV}$. For the lower energy $<100 \mathrm{MeV}$ the comprehensive investigations are required. In this article the various variants of superconducting options from $3 \mathrm{MeV}$ up to $40-100 \mathrm{MeV}$ are discussed. The considered variants include both the conventional combination of half-wave and spoke cavity with quadrupoles and new schemes. In conclusion major parameters for different structures are discussed.
\end{abstract}

Contribution to the PAC05, Knoxville, USA

Work supported by the European Community-Research Infrastructure Activity under the FP6 "Structuring the European Research Area” programme (CARE, contract number RII3-CT2003-506395). 


\title{
COMPARISON OF BEAM DYNAMIC IN DIFFERENT SUPERCONDUCTING OPTIONS OF LOW ENERGY HIGH INTENSE LINAC*
}

\author{
N.Vasyukhin, Yu.Senichev and R.Tölle, Forschungszentrum Jülich, Germany.
}

\begin{abstract}
At present the superconducting proton linacs have obvious applications in energy range $\sim 100-1000 \mathrm{MeV}$. For the lower energy $<100 \mathrm{MeV}$ the comprehensive investigations are required. In this article the various variants of superconducting options from $3 \mathrm{MeV}$ up to 40 $100 \mathrm{MeV}$ are discussed. The considered variants include both the conventional combination of half-wave and spoke cavity with quadrupoles and new schemes. In conclusion major parameters for different structures are discussed.
\end{abstract}

\section{INTRODUCTION}

The room-temperature solution for the low energy part of high intensity linear accelerator is well studied and successfully used for the pulsed regime. However, for $\mathrm{CW}$ mode the power consumption and heating problems lead to a necessity to use super-conducting structure, but not the normal-conducting one.

The super-conducting linear accelerators have a number of advantages in comparison with the room temperature accelerators: high accelerating gradient, low energy losses and high duty cycle. But despite of these benefits there are some problems, which are common with normalconducting machines. In particular, it is well know in the case of space charge dominated beam the linear detuning of space charge can reach $50 \%$, and the compensation of linear detuning by the external focusing elements moves the halo out of the stable region due to the non-linear detuning of these particles. Another common strongest restriction is the fixed loss levels, about $5 \mathrm{~W} / \mathrm{m}$.

Although, in the super-conducting cavity the large aperture is allowed due to the absence of high shunt impedance requirement, and the main part of losses is usually located in quadrupoles, which aperture is much larger.

Taking into account all these features, few conceptual schemes for super-conducting low energy part of high intensity proton linear accelerator were considered. The point of this article to give conclusion which scheme is more preferable from beam dynamics point of view.

\section{FIRST SCHEME}

As first scheme we consider the super-conducting option with external focusing quadrupoles. Since the cold-warm transitions takes a space the number of accelerating gaps should be increased in order to get the higher real estate gradient. It causes the relatively long

*Work supported by the European Community-Research Infrastructure Activity under the FP6 "Structuring the European Research Area" program (CARE, contract number RII3-CT-2003-506395) focusing period and the stronger modulation of beta function. Simultaneously, in the case of high intensity beams the long focusing period leads to the higher space charge effect in the radial motion, which one leads to the various resonances and the emittance increasing. In this scheme the type of structure does not play a decisive role for the beam dynamics. However, in the same time there are a lot of specific features in electrodynamics for each cavity type. For performed simulations the peak electric field was considered to be $\sim 40 \mathrm{MV} / \mathrm{m}$, the peak magnetic field $\sim 80 \mathrm{mT}$. The synchronous phase was chosen $-30^{\circ}$ in order to provide the stable motion in the longitudinal plane for the high intensity beams.

\section{Eight accelerating gaps per focusing period}

As an example for the first option of a superconducting accelerator the design of an injector-linear accelerator for COSY ring was taken [1]. The focusing period consists of four super-conducting half-wave cavities [2] in one cryo-module and an external quadrupole doublet. From our simulation with space charge we found out the maximum current in this design is about $30 \mathrm{~mA}$ with losses of order $5 \mathrm{~W} / \mathrm{m}$ and a duty factor of $100 \%$. Over 11 periods the proton beam is accelerated from $2.5 \mathrm{MeV}$ up to $50 \mathrm{MeV}$. The first 5 periods operate at $160 \mathrm{MHz}$ and the next 6 ones operate at $320 \mathrm{MHz}$. The transverse RMS sizes are shown on figure 1.

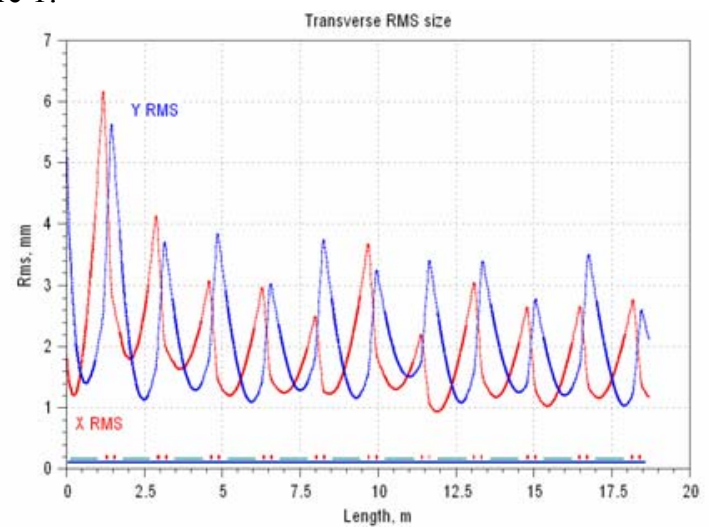

Figure 1: Transverse RMS vs. linac length

In the considered option with eight gaps it is difficult to match the beam due to the space charge tune shift $40 \%$, and the required level of losses determines the maximum current $30 \mathrm{~mA}$. As consequence the transverse RMS emittance growth for this case is about $40 \%$.

\section{Four accelerating gaps per focusing period}

In order to increase the maximum current the focusing period should be shorter, at least, for the initial part of 
acceleration. The could-warm transition is already minimized. Therefore only number of accelerating gaps can be decreased. As an example the beam dynamics in accelerating channel with four accelerating gaps was simulated, using the same cavities. In the four gaps option the focusing period length is $\sim 1.2 \mathrm{~m}$, and the real estate gradient decreases to $\sim 1.7 \mathrm{MV} / \mathrm{m}$ from $\sim 2.5 \mathrm{MV} / \mathrm{m}$. With lower number of accelerating gaps the ratio between the linac's parts with and without accelerating field decreases, and the shape of separatrix significantly changes (see figure 2).

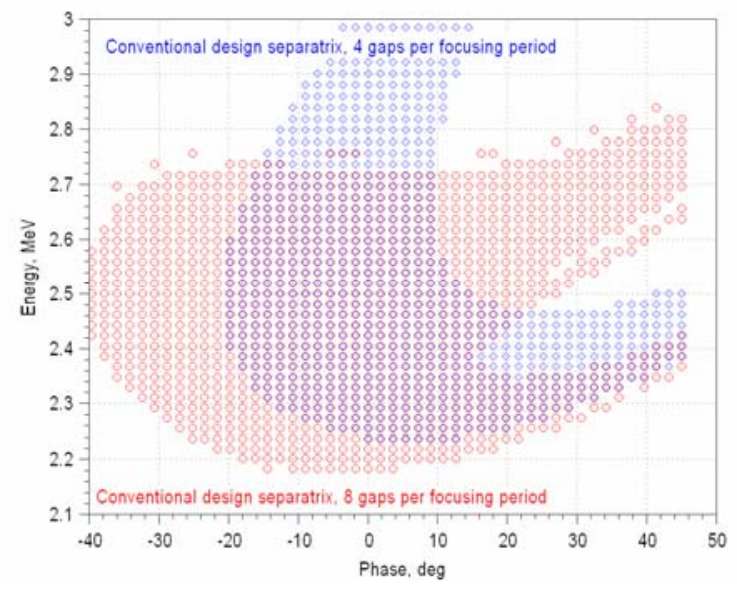

Figure 2: Longitudinal separatrix for eight and four accelerating gaps per focusing period

Therefore in order to match the beam in the longitudinal plane the initial pre-bunching of the beam is needed. For the simulation procedure we just took the bunch length $\pm 7.5^{\circ}$ instead of $\pm 15^{\circ}$ as for first option to simplify the calculations.

In result of simulation it was found

- $\quad$ in spite of the longitudinal mismatching due to the shorter focusing period the maximum current limit rose up to $60 \mathrm{~mA}$

- $\quad$ in the longitudinal plane the motion remains to be stable, but the bunch shape undergoes a modification.

\section{SECOND SCHEME}

As second scheme we considered the linear accelerator with the internal focusing elements inside of cry-modules. This solution is technically complicated, but obviously it promises to be a good candidate for acceleration of high intensity beams. If the focusing elements either solenoids, or quadrupoles could be placed in cryo-modules the place of the cold-warm transition can be saved, and the focusing period length becomes shorter. In particular, the simulated four accelerating gaps option has the focusing period length $0.85 \mathrm{~m}$.

We have considered the first 20 cavities, since the most of the problems are appeared at the beginning of acceleration. During our simulation it was found out that the maximum current limit rose up to $160 \mathrm{~mA}$ with the same level of losses. But the shorter focusing period is not the only reason for the higher current limit, since in this option the parametrical resonance in longitudinal plane is avoided as well. However, from the technical point of view this scenario is unpredictably difficult due to absence of the technical solution how to place the quadrupoles (or strong solenoids) inside cryo-modules. The transverse RMS sizes are shown (see fig. 3).

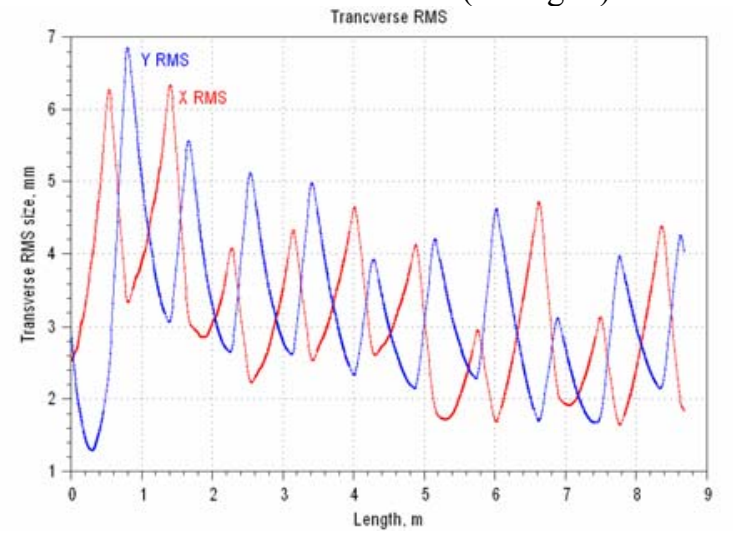

Figure 3: Transverse RMS vs. Linac Length

The envelopes behaviour seems to be mismatched, but as in the first case the quadrupoles gradients were optimised to get the maximum current within the certain level of losses.

\section{THIRD SCHEME}

As last option we consider the scheme with RFfocusing by electro-magnetic field in the cavity. This type of RF focusing is studied in the normal-conducting solution for the low energy part of linear accelerator [34]. In such type of structures the focusing is provided by the insertion of 4 cylindrical electrodes in the gap. The distance between focusing electrodes defines focusing strength as well as aperture size. Therefore in this option aperture cannot be as large as in the conventional design. Due to the RF requirements the geometry of superconductive cavity has to be simplified as much as possible. Also cavities belonging to one family should have the identical geometry. Therefore in such accelerators the longitudinal and transverse dynamics of high intense beam are more complicated to describe.

The accelerating channel based on RF-focusing was simulated and the beam dynamics investigations were performed. During simulations the average real estate gradient was achieved $\sim 1.7 \mathrm{MV} / \mathrm{m}$. The total normalized transverse acceptance for $352 \mathrm{MHz}$ option is around $4 \cdot \pi \cdot \mathrm{mm} \cdot \mathrm{mrad}$. Because of focusing by RF-field the longitudinal motion is not perturbed by the drifts and longitudinal phase portrait of high intensity beam conserves the elliptical shape (see fig. 4). 


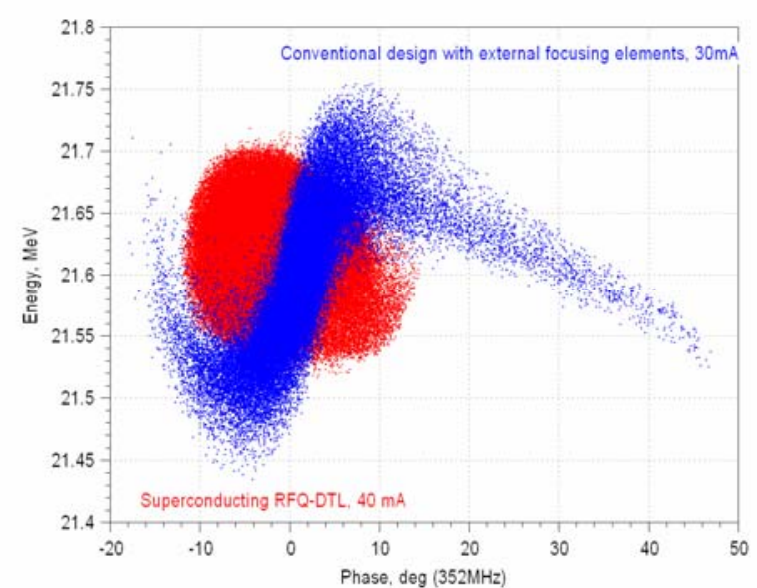

Figure 4: Longitudinal phase portraits

During simulation it was investigated that the maximum current limit is up to $40 \mathrm{~mA}$ for this option with the same level of losses.

\section{CONCLUSIONS}

Thus, one can conclude that the most attractive superconductive option for the low energy part linear accelerator from the beam dynamic point of view is the scheme with superconducting focusing elements in cryomodule. This option allows accelerating the high currents in $\mathrm{CW}$ mode with quite high real estate gradient. But this option is complicated technically.

The conventional design with the room-temperature quadrupoles provides the lower real estate gradient, and simultaneously the drifts for cold-warm transitions perturbs longitudinal motion leading to the non-elliptical bunch shape in the longitudinal plane for the high intensity beams. The maximum current mostly depends on the length of focusing period and varies in the range 30-60mA.

The beam dynamics studies for the scheme with RFfocusing shows that this option can be used for acceleration of up to $40 \mathrm{~mA}$ protons current with high enough real estate gradient and smooth longitudinal motion. The advantages of this option are that all cavities can be installed in one cryo-module, the quadrupoles are not used, and the smooth longitudinal motion do not perturbs the bunch shape.

\section{ACKNOWLEDGEMENTS}

Authors would like to thank Dr. Prof. R. Maier for support and scientific discussions.

\section{REFERENCES}

[1] R.Tölle et al., "A superconducting injector linac for COSY” EPAC 02, Paris, 2002, p. 966

[2] http://www.fz-juelich.de/ikp/publications/ AR2003/CHAP6/607.pdf

[3] V.A. Teplyakov, Pribory \& Technika Experimenta, 1964, v.6, p. 24

[4] Yu.A. Budanov et al., "RFQ drift-tube proton linacs in IHEP”, LINAC'04, Lübeck, August 2004, p. 285. 